\title{
Bernoulli Related Polynomials and Numbers
}

\author{
By Ch. A. Charalambides
}

Abstract. The polynomials $\varphi_{n}(x ; a, b)$ of degree $n$ defined by the equations

$$
\Delta_{a} \varphi_{n}(x ; a, b)=\frac{(x)_{n-1, b}}{b^{n-1} \cdot(n-1) !} \text { and } \Delta_{b} \varphi_{n}(x ; a, b)=\varphi_{n-1}(x ; a, b)
$$

where $(x)_{n, b}=x(x-b)(x-2 b) \cdots(x-n b+b)$ is the generalized factorial and $\Delta_{a} f(x)=f(x+a)-f(x)$, are the subject of this paper. A representation of these polynomials as a sum of generalized factorials is given. The coefficients, $B(n, s)$, $s=a / b$, of this representation are given explicitly or by a recurrence relation. The generating functions of $\varphi_{n}(x ; a, b)$ and $B(n, s)$ are obtained. The limits of $\varphi_{n}(x ; a, b)$ as $a \rightarrow 1, b \rightarrow 0$ or $a \rightarrow 0, b \rightarrow 1$ and the limits of $B(n, s)$ as $s \rightarrow$ $\pm \infty$ or $s \rightarrow 0$ are shown to be the Bernoulli polynomials and numbers of the first and second kind, respectively. Finally, the generalized factorial moments of a discrete rectangular distribution are obtained in terms of $B(n, s)$ in a form similar to that giving its usual moments in terms of the Bernoulli numbers.

1. Definitions and General Results. Let $\Delta_{a}$ denote the difference operator defined by $\Delta_{a} f(x)=f(x+a)-f(x)$ and $(x)_{n, b}$ the generalized factorial of degree $n$ defined by $(x)_{n, b}=x(x-b)(x-2 b) \cdots(x-n b+b)$. Let $\varphi_{n}(x ; a, b)$ be a polynomial of degree $n$ satisfying the following equation

$$
\Delta_{a} \varphi_{n}(x ; a, b)=\frac{(x)_{n-1, b}}{b^{n-1} \cdot(n-1) !}=b^{-n+1}\left(\begin{array}{c}
x \\
n-1
\end{array}\right)_{b} .
$$

From this equation and since

$$
\Delta_{b}\left(\begin{array}{c}
x \\
n-1
\end{array}\right)_{b}=b\left(\begin{array}{c}
x \\
n-2
\end{array}\right)_{b}
$$

it follows that

$$
\Delta_{b} \Delta_{a} \varphi_{n}(x ; a, b)=\Delta_{a} \varphi_{n-1}(x ; a, b) .
$$

Performing the operation $\Delta_{a}^{-1}$ on both members of this equation and noting that the operations $\Delta_{a}$ and $\Delta_{b}$ are commutative, we obtain

$$
\Delta_{b} \varphi_{n}(x ; a, b)=\varphi_{n-1}(x ; a, b) .
$$

Received December 15, 1977 ; revised August 21, 1978.

AMS (MOS) subject classifications (1970). Primary 10A40, Secondary 62E99.

Key words and phrases. Difference operator, generalized factorial, Stirling polynomials, Stirling numbers of the first and second kind, Bernoulli polynomials and numbers of the first and second kind, generating functions, probability factorial moments. 
Since we are dealing with polynomials only, this solution is univocal (see for example Jordan [7, p. 102]) and the polynomials $\varphi_{n}(x ; a, b)$ are completely determined by the equations (1.1) and (1.2).

The expansion of the polynomial into a Newton series may be written as

$$
\varphi_{n}(x ; a, b)=\sum_{m=0}^{n} c_{m} b^{-n+m}\left(\begin{array}{c}
x \\
n-m
\end{array}\right)_{b}
$$

where the coefficients are independent of the degree of the polynomial. To determine them we may proceed as follows: From (1.1) we deduce $\Delta_{a} \varphi_{1}(x ; a, b)=1$; and hence, $\varphi_{1}(x ; a, b)=x / a+c_{1}$ so that $c_{0}=1 / s, s=a / b$. For $n>1$ we have $\Delta_{a} \varphi_{n}(0 ; a, b)=0$. On the other hand, we have from (1.3)

$$
\Delta_{a} \varphi_{n}(0 ; a, b)=\sum_{m=0}^{n-1} c_{m} b^{-n+m}\left[\Delta_{a}\left(\begin{array}{c}
x \\
n-m
\end{array}\right)_{b}\right]_{x=0} .
$$

Since

$$
\left[\Delta_{a}\left(\begin{array}{c}
x \\
n-m
\end{array}\right)_{b}\right]_{x=0}=\left[\left(\begin{array}{c}
x+a \\
n-m
\end{array}\right)_{b}-\left(\begin{array}{c}
x \\
n-m
\end{array}\right)_{b}\right]_{x=0}=\left(\begin{array}{c}
a \\
n-m
\end{array}\right),
$$

we get

$$
\sum_{m=0}^{n-1} c_{m} b^{-n+m}\left(\begin{array}{c}
a \\
n-m
\end{array}\right) b=0
$$

or

$$
\sum_{m=0}^{n-1} c_{m}\left(\begin{array}{c}
s \\
n-m
\end{array}\right)=0, \quad s=a / b
$$

Starting from $c_{0}=1 / s$ we may determine by aid of (1.4), step by step, the coefficients $c_{m}, m=1,2, \ldots$. We note that these coefficients depend only on the ratio $a / b$ and not explicitly on $a$ and $b$. From (1.4) we obtain $c_{1}=-(s-1) / 2 s, c_{2}=$ $(s-1)(s+1) / 12 s$ and so on.

Introducing the numbers $B(m, s)=m ! c_{m}$, Eq. (1.3) may be written as

$$
\varphi_{n}(x ; a, b)=\frac{1}{n !} \sum_{m=0}^{n}\left(\begin{array}{l}
n \\
m
\end{array}\right) B(m, s)(x / b)_{n-m}
$$

or symbolically as

$$
\varphi_{n}(x ; a, b)=\frac{1}{n !}((x / b)+B(, s))^{n}, \quad(x / b)^{k} \equiv(x / b)_{k}, \quad B^{m}(, s) \equiv B(m, s) .
$$

Performing the operation $\Delta_{a}^{-1}$ if $n>1$ and since $\Delta_{a} \varphi_{n}(0 ; a, b)=0$ we get the symbolic formula

$$
((s)+B(, s))^{n}-B(n, s)=0
$$


giving the numbers $B(n, s)$. Starting from $B(0, s)=1 / s$, we may obtain $B(1, s)=$ $-(s-1) / 2 s, B(2, s)=(s-1)(s+1) / 6 s$ and so on.

A second expansion of the polynomial $\varphi_{n}(x ; a, b)$ into a series of generalized factorials $(x)_{k, a}$ may be obtained as follows: Using the expansion (see [4]),

$$
(x)_{n-1, b}=\sum_{k=0}^{n-1} b^{n-1} \cdot a^{-k} C(n-1, k, s)(x)_{k, a}, \quad s=a / b,
$$

(1.1) may be written as

$$
\Delta_{a} \varphi_{n}(x ; a, b)=\frac{1}{(n-1) !} \sum_{k=0}^{n-1} C(n-1, k, s) a^{-k}(x)_{k, a}
$$

Since

$$
\Delta_{a}^{-1}(x)_{k, a}=\frac{(x)_{k+1, a}}{a(k+1)}+K
$$

we obtain

$$
\varphi_{n}(x ; a, b)=\frac{1}{(n-1) !} \sum_{k=0}^{n-1} C(n-1, k, s) \frac{(x)_{k+1, a}}{(k+1) a^{k+1}}+K .
$$

But $\varphi_{n}(0 ; a, b)=B(n, s) / n !$ and hence $K=B(n, s) / n$ ! so that

$$
\varphi_{n}(x ; a, b)=\frac{1}{(n-1) !} \sum_{k=0}^{n-1} C(n-1, k, s) \frac{(x)_{k+1, a}}{(k+1) a^{k+1}}+\frac{B(n, s)}{n !} \text {. }
$$

From (1.7) it follows that

$$
\Delta_{a}^{m} \varphi_{n}(0 ; a, b)=\frac{(m-1) !}{(n-1) !} C(n-1, m-1, s)
$$

and

$$
\varphi_{n}(0 ; a, b)=\varphi_{n}(a ; a, b)=B(n, s) / n !
$$

Using (1.7), we may determine the numbers $B(m, s)$ in terms of the numbers $C(n, k, s)$. From (1.7) and the relation

$$
(x)_{k+1, a}=\sum_{m=0}^{k+1} a^{k+1} b^{-m} C(k+1, m, r)(x)_{m, b}, \quad r=b / a=1 / s,
$$

we have

$$
\begin{aligned}
\varphi_{n}(x ; a, b)= & \frac{1}{(n-1) !} \sum_{k=0}^{n-1} C(n-1, k, s) \frac{1}{k+1} \sum_{m=0}^{k+1} C(k+1, m, r) b^{-m}(x)_{m, b} \\
& +\frac{B(n, s)}{n !}
\end{aligned}
$$


or

$$
\begin{aligned}
\varphi_{n}(x ; a, b)= & \sum_{m=0}^{n-1} \frac{1}{(n-1) !} \sum_{k=m-1}^{n-1}\left\{\frac{1}{k+1} C(n-1, k, s) C(k+1, m, r)\right\} b^{-m}(x)_{m, b} \\
& +\frac{B(n, s)}{n !}
\end{aligned}
$$

Comparing the last relation with (1.5), we get

$$
B(n-m, s)=n\left[\left(\begin{array}{c}
n \\
m
\end{array}\right)\right]^{-1} \sum_{k=m-1}^{n-1} \frac{1}{k+1} C(n-1, k, s) C(k+1, m, r), \quad r s=1 .
$$

Putting $m=1$ and writing $n+1$ instead of $n$ and since $C(k+1,1, r)=(r)_{k+1}$, we finally obtain

$$
B(n, s)=\sum_{k=0}^{n} \frac{(r)_{k+1}}{k+1} C(n, k, s)
$$

which on using (see [3]) the relation

$$
C(n, k, s)=\frac{1}{k !} \sum_{m=0}^{k}(-1)^{k-m}\left(\begin{array}{l}
k \\
m
\end{array}\right)(s m)_{n}
$$

may be written as

$$
B(n, s)=\sum_{k=0}^{n} \sum_{m=0}^{k}(-1)^{k-m}\left(\begin{array}{c}
r \\
k+1
\end{array}\right)\left(\begin{array}{l}
k \\
m
\end{array}\right)(s m)_{n}, \quad r s=1
$$

2. Generating Functions. Consider first the problem of expanding a polynomial $f(x)$ of degree $n$ into a series of polynomials $\varphi_{i}(x ; a, b), i=0,1, \ldots, n$, and let

$$
f(x)=\sum_{i=0}^{n} k_{i} \varphi_{i}(x ; a, b) .
$$

Since $\Delta_{b}^{m} \varphi_{n}(x ; a, b)=\varphi_{n-m}(x ; a, b)$,

$$
\begin{aligned}
\Delta_{b}^{m} f(x) & =k_{m} \varphi_{0}(x ; a, b)+\sum_{i=m+1}^{n} k_{i} \varphi_{i-m}(x ; a, b) \\
& =k_{m} \cdot b / a+\sum_{i=m+1}^{n} k_{i} \varphi_{i-m}(x ; a, b), \quad m=0,1,2, \ldots
\end{aligned}
$$

Performing the operation $\Delta_{b}^{-1}$ on both members of this equation, we obtain

$$
\Delta_{b}^{m-1} f(x)=k_{m} \cdot x / a+\sum_{i=m+1}^{n} k_{i} \varphi_{i-m+1}(x ; a, b)+K
$$

and since $\varphi_{n}(0 ; a, b)=\varphi_{n}(a ; a, b)$,

$$
k_{m}=\left[\Delta_{b}^{m-1} f(x)\right]_{x=a}-\left[\Delta_{b}^{m-1} f(x)\right]_{x=0}=\left[\Delta_{a} \Delta_{b}^{m-1} f(x)\right]_{x=0} .
$$


When $f(x)$ is not a polynomial then the series (2.1) will be infinite and considerations of convergence must be made. But the coefficients $k_{m}$ will still be given by (2.2).

Let us now determine the generating function $f(t ; x)$ of the polynomials $\varphi_{m}(x ; a, b)$. In this case we must have

$$
k_{m}=\left[\left.\Delta_{a} \Delta_{b}^{m-1} f(t ; x)\right|_{t=0}=t^{m}, \quad m=0,1,2, \ldots .\right.
$$

Since $\Delta_{b}^{m-1}(1+t)^{x / b}=t^{m-1}(1+t)^{x / b}$ and $\Delta_{a}(1+t)^{x / b}=(1+t)^{x / b}\left[(1+t)^{s}-1\right]$, we get

$$
k_{m}=\left[\Delta_{a} \Delta_{b}^{m-1} \frac{t(1+t)^{x / b}}{(1+t)^{s-1}}\right]_{x=0}=t^{m}
$$

Therefore,

$$
\sum_{m=0}^{\infty} \varphi_{m}(x ; a, b) t^{m}=\frac{t(1+t)^{x / b}}{(1+t)^{s}-1} .
$$

Putting $x=0$, we obtain the exponential generating function of the numbers $B(m, s)$

$$
\sum_{m=0}^{\infty} B(m, s) \frac{t^{m}}{m !}=\frac{t}{(1+t)^{s}-1} .
$$

The exponential generating function (2.4) may be expanded in the following way:

$$
\begin{aligned}
\frac{t}{(1+t)^{s}-1} & =\frac{1 / s}{1+\frac{1}{s}\left[\frac{(1+t)^{s}-1}{t}-s\right]}=\sum_{n=0}^{\infty} \frac{(-1)^{n}}{s^{n+1}}\left[\frac{(1+t)^{s}-1}{t}-s\right]^{n} \\
& =\frac{1}{s}+\sum_{n=1}^{\infty} \frac{(-1)^{n}}{s^{n+1}}\left[\sum_{k=2}^{\infty}\left(\begin{array}{l}
s \\
k
\end{array}\right) t^{k-1}\right]^{n} \\
& =\frac{1}{s}+\sum_{m=1}^{\infty}\left[\sum_{n=1}^{m} \frac{(-1)^{n}}{s^{n+1}} \prod_{i=1}^{n}\left(\begin{array}{c}
s \\
k_{i}
\end{array}\right)\right] t^{m},
\end{aligned}
$$

where $k_{i} \geqslant 2, i=1,2, \ldots, n, k_{1}+k_{2}+\cdots+k_{n}=m-n$. Therefore, we conclude that

$$
B(m, s)=m ! \sum_{n=1}^{m} \frac{(-1)^{n}}{s^{n+1}} \prod_{i=1}^{n}\left(\begin{array}{c}
s \\
k_{i}
\end{array}\right), \quad k_{i} \geqslant 2, i=1,2, \ldots, n, \quad \sum_{i=1}^{n} k_{i}=m-n .
$$

3. Limits. Using the relations (see [3])

$$
\lim _{s \rightarrow 0} C(n, k, s) / s^{k}=s(n, k), \quad \lim _{s \rightarrow \pm \infty} C(n, k, s) / s^{n}=S(n, k),
$$

we get from (1.9)

$$
\begin{gathered}
\lim _{s \rightarrow 0} s B(n, s)=\sum_{k=0}^{n} \frac{s(n, k)}{k+1}=n ! b_{n}, \\
\lim _{s \rightarrow \pm \infty} s^{-n+1} B(n, s)=\sum_{k=0}^{n} \frac{(-1)^{k}}{k+1} k ! S(n, k)=B_{n},
\end{gathered}
$$


where $B_{n}$ and $b_{n}$ denote the Bernoulli numbers of the first and second kind, respectively (see Jordan [7, pp. 236, 267]).

Similarly from (1.7) we may obtain

$$
\lim _{\substack{a \rightarrow 0 \\ b \rightarrow 1}} a \varphi_{n}(x ; a, b)=\frac{1}{(n-1) !} \sum_{k=0}^{n} s(n-1, k) \frac{x^{k+1}}{k+1}+b_{n}=\psi_{n}(x)
$$

and

$$
\lim _{\substack{a \rightarrow 1 \\ b \rightarrow 0}} b^{-n+1} \varphi_{n}(x ; a, b)=\frac{1}{(n-1) !} \sum_{k=0}^{n} S(n-1, k) \frac{(x)_{k+1}}{k+1}+\frac{B_{n}}{n !}=\varphi_{n}(x),
$$

where $\varphi_{n}(x)$ and $\psi_{n}(x)$ denote the Bernoulli polynomials of the first and second kind, respectively.

4. The $C$ and the Stirling Polynomials and the Numbers $B(n, s)$. It was shown in [4] that the $C$-number $C(x, x-n, s) / s^{x-n}$ is a polynomial of $x$ of degree $2 n$

$$
C(x, x-n, s) / s^{x-n}=\sum_{k=0}^{n-1} D(n, k, s-1)\left(\begin{array}{c}
x \\
2 n-k
\end{array}\right)
$$

with the coefficients satisfying the recurrence relation

$$
D(n, k, s)=(2 n-k+1) s D(n, k, s)+[(n-k+1) s-n] D(n, k-1, s)
$$

with $D(1,0, s)=s$ and $D(n, k, s)=0$ if $k>n-1$.

Following Nielsen (see Jordan [7, p. 224]), let us call a $C$-polynomial the expression

$$
c_{n}(n-x-1 ; s)=\frac{(-1)^{n} C(x+1, x-n, s)}{s^{x-n} \cdot(x+1)_{n+2}}, \quad x>n,
$$

which on using (4.1) may be written as

$$
c_{n}(n-x-1 ; s)=(-1)^{n} \sum_{k=0}^{n} D(n+1, k, s-1) \frac{(x-n-1)_{n-k}}{(2 n-k+2) !} .
$$

The generating function of $c_{n}(x ; s)$ may be obtained as follows: From (4.3) we have

$$
c_{n}(n-m ; s)=\frac{(-1)^{n} C(m, n-n-1, s)}{s^{m-n-1} \cdot(m)_{n+2}}
$$

and putting $n=m-x-1$ we get

$$
c_{m-x-1}(-x-1 ; s)=(-1)^{m-x-1} \cdot s^{-x} C(m, x, s)(x-1) ! / m !
$$

or

$$
\frac{x !}{m !} C(m, x, s)=(-1)^{m-x-1} \cdot x s^{x} c_{m-x-1}(-x-1 ; s)
$$

Multiplying by $t^{m}$ and summing for $m=x+1, x+2, \ldots$, we get 


$$
\left[(1+t)^{s}-1\right]^{x}-s^{x} t^{x}=x s^{x} \sum_{m=x+1}^{\infty}(-1)^{m-x-1} c_{m-x-1}(-x-1 ; s) t^{m}
$$

Dividing by $x s^{x} t^{x+1}$ we obtain

$$
\frac{(-1)^{x}\left[1-(1+t)^{s}\right]^{x}}{x s^{x} t^{x+1}}-\frac{1}{x t}=\sum_{m=0}^{\infty}(-1)^{m} c_{m}(-x-1 ; s) t^{m} .
$$

Putting $-t$ instead of $t$ and $x$ instead of $-x-1$, we finally get

$$
\sum_{m=0}^{\infty} c_{m}(x ; s) t^{m}=\frac{s^{x+1} \cdot t^{x}}{(x+1)\left[1-(1-t)^{s}\right]^{x+1}}-\frac{1}{(x+1) t}
$$

From (4.4) and using (4.2) we may obtain for the polynomials $c_{m}(x ; s)$ the recurrence

$$
[(s-1)(x+1)+n] c_{n-1}(x ; s)=(n-x) c_{n}(x ; s)+(x+2) c_{n}(x+1 ; s)
$$

with $c_{0}(x ; s)=(s-1) s^{x+1} / 2$.

If we put $x=0$ into the generating function (4.5), we have

$$
\sum_{m=0}^{\infty} c_{m}(0 ; s) t^{m}=\frac{s t}{1-(1-t)^{s}}-\frac{1}{t}
$$

or

$$
\frac{1}{s}+\sum_{m=1}^{\infty}(-1)^{m} \frac{c_{m-1}(0 ; s)}{s} \cdot t^{m}=\frac{t}{(1+t)^{s}-1}
$$

The second member of this relation is the exponential generating function (2.4) of the numbers $B(m, s)$; therefore, we have

$$
c_{m-1}(0 ; s)=\frac{(-1)^{m} s}{m !} B(m, s) .
$$

Using the relation $\lim _{s \rightarrow \pm \infty} C(n, k, s) / s^{n}=S(n, k)$, we may obtain from (4.3)

$$
\lim _{s \rightarrow \pm \infty} c_{n}(x ; s) / s^{n+1}=S_{n}(x)
$$

where $S_{n}(x)$ is the Stirling polynomial (see Jordan [7, p. 228]).

Similarly, using the relations

$$
\lim _{s \rightarrow 0} C(n, k, s) / s^{k}=s(n, k), \quad \lim _{s \rightarrow 0} C(n, k,-s) /(-s)^{k}=s(n, k),
$$

we get from (1.3)

$$
\lim _{s \rightarrow 0} c_{n}(n-x-1 ; s)=-S_{n}(x)
$$


5. Applications. From (1.1) and (1.5) we obtain

$$
\Delta_{a}^{-1} b^{-n}\left(\begin{array}{l}
x \\
n
\end{array}\right)_{b}=\varphi_{n+1}(x ; a, b)+K=\frac{1}{(n+1) !}((x / b)+B(, s))^{n+1}+K
$$

and hence,

$$
\sum_{x=0}^{a z} b^{-n}\left(\begin{array}{l}
x \\
n
\end{array}\right)_{b}=\frac{1}{(n+1) !}\left[((s z)+B(, s))^{n+1}-B(n+1, s)\right],
$$

where the $a$ in the symbol of the sum indicates that the summation is taken for equidistant values of $x$ with increment $a$.

Using this formula, we may determine the sum $\underset{\substack{a=0 \\ \sum_{a}}}{a z} f(x)$ of the function $f(x)$ which is given by its differences $\Delta_{b}^{n} f(0)$

$$
f(x)=\sum_{n=0}^{\infty} b^{-n}\left(\begin{array}{l}
x \\
n
\end{array}\right)_{b} \Delta_{b}^{n} f(0)
$$

Performing on both members of this relation, the operation $\Delta_{a}^{-1}$, we get

$$
\Delta_{a}^{-1} f(x)=\sum_{n=0}^{\infty} \varphi_{n+1}(x ; a, b) \Delta_{b}^{n} f(0)+K
$$

and finally,

$$
\sum_{x=0}^{a z} f(x)=\sum_{n=0}^{\infty} \frac{1}{(n+1)}\left[((s z)+B(, s))^{n+1}-B(n+1, s)\right] \Delta_{b}^{n} f(0) .
$$

As a special case of (5.1), let $a=b=1$. Since $B(0,1)=1, B(n, 1)=0$ if $n>$ 0 we obtain the well-known formula $\Sigma_{x=0}^{z}\left(\begin{array}{l}x \\ n\end{array}\right)=\left(\begin{array}{c}z \\ n+1\end{array}\right)$.

A useful formula may be obtained from (5.2) if we take $f(x)=(x)_{-m, b}$. Since $\Delta_{b}^{n}(x)_{-m, b}=b^{n}(-m)_{n}(x)_{-m-n, b}$, we get from (5.2)

$$
\sum_{x=0}^{a z}(x)_{-m, b}=\frac{(-1)^{m} b^{m}}{m+1}\left[((s z)+B(, s))^{m+1}-B(m+1, s)\right] .
$$

In statistical theory the $n$th factorial moment about an arbitrary origin $c$ may be defined by the equation (see e.g. Kendall and Stuart $[8$, p. 63])

$$
\mu_{(n ; b)}^{\prime}(c)=\sum_{j}\left(x_{j}-c\right)_{n, b} P\left(X=x_{j}\right)
$$

where $(x)_{n, b}=x(x-b) \cdots(x-n b+b)$ is the generalized factorial of degree $n$. They are used almost entirely for discrete distributions or continuous distributions grouped in intervals of width $b$. Formula (5.1) may be used for expressing the factorial moments $\mu_{(n ; b)}^{\prime}=\mu_{(m ; b)}^{\prime}(0)$ about $c=0$ of the discrete rectangular distribution with probability function

$$
P(X=x)=1 / N, \quad x=a, 2 a, \ldots, N a .
$$


From (5.3) and using (5.1), we get

$$
\mu_{(n ; b)}^{\prime}=\frac{b^{n}}{N(n+1)}\left[((N s)+B(, s))^{n+1}-B(n+1, s)\right] .
$$

The factorial moments $\mu_{(n ; b)}^{\prime}(c)$ may be obtained from (5.5) using the formula

$$
\mu_{(n ; b)}^{\prime}(c)=\sum_{k=0}^{n}\left(\begin{array}{l}
n \\
k
\end{array}\right)(-c)_{k} \mu_{(k ; b)}^{\prime}
$$

The factorial moment generating function $G(t)=\Sigma_{n=0}^{\infty} \mu_{(n ; b)}^{\prime} t^{n} / n !$ of the distribution (5.4) may be obtained from the frequency generating function

$$
P(t)=\sum_{x=a}^{N a} \frac{1}{N} t^{x}=\frac{1}{N} \cdot \frac{t^{a}-t^{a(N+1)}}{1-t^{a}}
$$

if we replace $t$ by $(1+t / b)^{1 / b}$ (cf. Kendall and Stuart [8, p. 66]). We get

$$
G(t)=\frac{1}{N}(1+t / b)^{s} \cdot \frac{1-(1+t / b)^{N s}}{1-(1+t / b)^{s}}, \quad s=a / b .
$$

The factorial cumulant generating function $K(t)=\Sigma_{n=0}^{\infty} \kappa_{(n ; b)}^{\prime} t^{n} / n$ ! is then given by

$$
K(t)=\log \frac{(1+t / b)^{N s}-1}{N s t / b}-\log \frac{(1+t / b)^{s}-1}{s t / b}+s \log (1+t / b),
$$

which can be expanded by using (2.3). Writing (2.3) in the form

$$
s \sum_{m=1}^{\infty} \varphi_{m}(a-b ; a, b) t^{m-1}=\frac{s(1+t)^{s-1}}{(1+t)^{s}-1}-\frac{1}{t}
$$

and integrating from 0 to $t$, we get

$$
s \sum_{m=1}^{\infty} \varphi_{m}(a-b ; a, b) t^{m} / m=\log \frac{(1+t)^{s}-1}{s t} .
$$

Hence,

$$
K(t)=s \sum_{m=1}^{\infty}\left\{N \varphi_{m}(N a-b ; N a, b)-\varphi_{m}(a-b ; a, b)+(-1)^{m-1}\right\} \frac{b^{-m} t^{m}}{m}
$$

and

$$
\begin{aligned}
\kappa_{(n ; b)}^{\prime} & =(n-1) ! s b^{-n}\left\{N \varphi_{n}(N a-b ; N a, b)-\varphi_{n}(a-b ; a, b)+(-1)^{n-1}\right\} \\
& =\frac{s b^{-n}}{n}\left\{N((N s-1)+B(., N s))^{n}+((s-1)+B(., s))^{n}+(-1)^{n-1}\right\} .
\end{aligned}
$$

For $a=b=1$ it reduces to

$$
\kappa_{(n)}^{\prime} \equiv \kappa_{(n ; 1)}^{\prime}=\frac{N}{n} \sum_{m=0}^{n}\left(\begin{array}{l}
n \\
m
\end{array}\right) B(m, N)(N-1)_{n-m}+\frac{(-1)^{n-1}}{n},
$$

which may be compared with the result obtained by David and Barton [5, p. 54]. 
In applied statistics when moments are calculated from a numerically specified distribution which is grouped, there is present a certain amount of approximations due to the fact that the frequencies are concentrated at the midpoints of intervals. The problem of eliminating the errors due to grouping has attracted the attention of several authors (see Kendall and Stuart [8] and the references therein). Abernethy [1] and Graig [6] gave a derivation of the corrections in the moments of a discrete variable. The corrections for the cumulants were expressed by Graig in terms of the Bernoulli numbers. The corrections for the factorial cumulants can be expressed in terms of the numbers $B(n, s)$. Let us suppose that $N$ consecutive values of a random variable (r.v.) are grouped in a frequency of width $a$. Following Graig, we write $x_{i}=$ $x+\epsilon$, where $x_{i}$ is the class mark of the $i$ th frequency class, for any true value, $x$, of the discrete r.v. included in this frequency and $\epsilon$ is a r.v. with probabilitv function

$$
P\left(\epsilon=\epsilon_{k}\right)=\frac{1}{N}, \quad \epsilon_{k}=\frac{a}{N}(k-(N+1) / 2), \quad k=1,2, \ldots, N .
$$

If $G_{x_{i}}(t)$ and $G_{x}(t)$ are the exponential generating functions of the calculated factorial moments $\bar{\mu}_{(n)}^{\prime}$ and the corrected factorial moments $\mu_{(n)}^{\prime}$, respectively, then

$$
G_{x_{i}}(t)=G_{x}(t) \sum_{k=1}^{N} \frac{1}{N}(1+t)^{\epsilon} k
$$

or

$$
G_{x_{i}}(t)=G_{x}(t)\left[\frac{1}{N}(1+t)^{-a(N-1) / 2 N} \cdot \frac{1-(1+t)^{a}}{1-(1+t)^{a / N}}\right] .
$$

Taking the logarithms of both sides and remembering that the logarithm of the factorial moment generating function is the factorial cumulant generating function, we get

$$
K_{x}(t)=K_{x_{i}}(t)-\left[\log \frac{(1+t)^{a}-1}{a t}-\log \frac{(1+t)^{a / N}-1}{a t / N}-\frac{a(N-1)}{2 N} \log (1+t)\right] \text {. }
$$

Using (5.6), we finally obtain for the corrections for the factorial cumulants

$$
\begin{aligned}
\kappa_{(n)}^{\prime} & =\bar{\kappa}_{(n)}^{\prime}-(n-1) ! a\left[\varphi_{n}(a-1 ; a, 1)-\frac{1}{N} \varphi_{n}(a-N ; a, N)+\frac{N-1}{2 N}(-1)^{n}\right] \\
& =\bar{\kappa}_{(n)}^{\prime}-\frac{a}{n}\left[((a-1)+B(., a))^{n}-\frac{1}{N}((a-N)+B(., a / N))^{n}+\frac{N-1}{2 N}(-1)^{n}\right] .
\end{aligned}
$$

Remark. The polynomials $\beta_{m}(\lambda)$ defined by Carlitz [2] by their exponential generating function

$$
\sum_{m=0}^{\infty} \beta_{m}(\lambda) \frac{t^{m}}{m !}=\frac{t}{(1+t / \lambda)^{1 / \lambda}-1}
$$

are closely related (for given $s$ ) to the numbers $B(m, s)$. Indeed, comparing (5.7) with (2.4), we get

$$
B(m, s)=s^{m-1} \beta_{m}\left(s^{-1}\right)
$$


Though our interest is mainly concentrated on statistical applications, it should be noted that using (5.8) and the results obtained by Carlitz we may infer for the $B(m, s)$ some interesting arithmetic properties analogous to those of the Bernoulli numbers.

Acknowledgments. I wish to thank the referee for his valuable comments towards revising this paper and for bringing to my attention the paper of Carlitz [2].

Statistical Unit

University of Athens

Panepistemiopolis, Athens 621, Greece

1. J. R. ABERNETHY, "On the elimination of the systematic errors due to grouping," Ann. Math. Statist., v. 4, 1933, pp. 263-277.

2. L. CARLITZ, "A degenerate Staudt-Clausen theorem," Arch. Math., v. 7, 1956, pp. 2833.

3. CH. A. CHARALAMBiDES, "A new kind of numbers appearing in the n-fold convolution of truncated binomial and negative binomial distributions," SIAM J. Appl. Math., v. 33, 1977, pp. 279-288.

4. CH. A. CHARALAMBIDES, "Some properties and applications of the differences of the generalized factorials," SIAM J. Appl. Math., v. 36, 1979.

5. F. N. DAVID \& D. E. BARTON, Combinatorial Chance, Griffin, London, 1962.

6. C. C. GRAIG, "Sheppard's corrections for a discrete variable," Ann. Math. Statist., v. 7, 1936, pp. 55-61.

7. CH. JORDAN, Calculus of Finite Differences, Chelsea, New York, 1960.

8. M. G. KENDALL \& A. STUART, The Advanced Theory of Statistics, Vol. 1, Hafner, New York, 1961. 